\title{
A role for genetic accommodation in evolution?
}

\author{
Christian Braendle ${ }^{1 *}$ and Thomas Flatt ${ }^{2}$
}

\begin{abstract}
Summary
Whether evolutionary change can occur by genetic assimilation, or more generally by genetic accommodation, remains controversial. Here we examine some of the experimental evidence for both phenomena. Several experiments in Drosophila suggest that assimilation is possible, and a new paper ${ }^{(1)}$ shows that a color polyphenism in the tobacco hornworm, Manduca sexta, can evolve by genetic accommodation. We argue that genetic accommodation, including assimilation, is a plausible mechanism in evolution; however, more work is required to test how this mechanism acts and how often it is involved in evolutionary change. BioEssays 28:868873, 2006. (c) 2006 Wiley Periodicals, Inc.
\end{abstract}

\section{Genetic assimilation and accommodation}

Whether the processes of genetic assimilation ${ }^{(2-9)}$ and accommodation ${ }^{(1,10)}$ can explain evolutionary change and phenotypic novelty is a controversial issue among evolutionary biologists (for example Refs 11-16).

In the 1940s and 1950s, Conrad Hal Waddington introduced the concept of genetic assimilation. ${ }^{(2-6)}$ Genetic assimilation is the evolutionary process by which a phenotype produced specifically in response to some environmental stimulus, such as a stressor, becomes stably expressed independently of the evoking environmental effect. ${ }^{(2-4,9)}$ How does this process of assimilation work? First, in the absence of an environmental stimulus, a particular threshold trait is stably expressed, and phenotypic deviants remain cryptic because the environmental threshold for their expression is too high. Second, in the presence of an environmental stimulus, previously cryptic genetic variation for the threshold trait is uncovered ${ }^{(2,17-20)}$ and the threshold for the expression of deviant phenotypes not seen under normal conditions is

\footnotetext{
${ }^{1}$ Institut Jacques Monod, CNRS, Universités Paris 6 and 7, Tour 43, Paris, France.

${ }^{2}$ Division of Biology and Medicine, Department of Ecology and Evolutionary Biology, Brown University, Providence, RI

Funding agency: Swiss National Science Foundation.

${ }^{*}$ Correspondence to: Christian Braendle, CNRS, Universités Paris 6 and 7, Institut Jacques Monod, Tour 43, 2 place Jussieu, 75251 Paris, France. E-mail: braendle @ijm.jussieu.fr

DOI 10.1002/bies.20456

Published online in Wiley InterScience (www.interscience.wiley.com).
}

lowered. Third, selection in the presence of the environmental factor enriches the previously cryptic alleles determining the trait. Eventually, these alleles become so frequent that the expression of the trait overcomes the higher threshold in the absence of the environmental stimulus. ${ }^{(9,20)}$ Thus, genetic assimilation transforms an environmentally induced (phenotypically plastic) trait into a phenotype which is stably expressed without the eliciting environmental stimulus: the genetically assimilated phenotype is no longer plastic, but exhibits a genetically fixed response independent of the environmental conditions, ${ }^{(2,9,14,16)}$ a phenomenon called canalization. ${ }^{(20)}$

Genetic assimilation is a special case of a more general phenomenon, called genetic accommodation, most prominently proposed by Mary Jane West-Eberhard in 2003. ${ }^{(10)}$ This scenario of phenotypic evolution posits that (1) a mutation or environmental change triggers the expression of a novel, heritable phenotypic variant, (2) the initially rare variant phenotype starts to spread (in the case of an environmentally induced change, due to the consistent recurrence of the environmental factor), creating a subpopulation expressing the novel trait, and (3) selection on existing genetic variation for the regulation or form of the trait causes it to become (a) genetically fixed or to remain (b) phenotypically plastic. ${ }^{(10)}$ Note that, in the strict sense, only process (3) represents genetic accommodation as it is defined by West-Eberhard, ${ }^{(10)}$ but, for the sake of conceptual simplicity, we refer here to genetic accommodation as the entire sequence of steps (1) to (3).

What then is the relationship between genetic assimilation and accommodation? Genetic assimilation describes only scenario (3a), i.e. the fixation of the response leading to environmental insensitivity, also called "environmental canalization," (10) whereas genetic accommodation can describe both the evolution of environmentally insensitive (3a) and sensitive (3b) trait expression. Another difference between the two concepts is that the model of genetic accommodation assumes that the trigger uncovering previously cryptic or novel phenotypes is either genetic or environmental, whereas the concept of genetic assimilation typically assumes only an environmental trigger. Thus, genetic accommodation is a generalization of genetic assimilation.

At least theoretically, genetic accommodation might thus facilitate phenotypic diversification under environmental or genetic change, adaptation to novel environments, 
and speciation. ${ }^{(10,20-23)}$ However, assimilation and accommodation remain controversial concepts, mainly because of a lack of convincing empirical evidence (for example Refs 1116) Here we review the experimental evidence for genetic assimilation in $\operatorname{Drosophila}^{(7,8)}$ and discuss the first clear experimental case of genetic accommodation of an environmentally sensitive phenotype from recent work on the tobacco hornworm, Manduca sexta. ${ }^{(1)}$

\section{Experimental evidence for genetic assimilation}

Several experiments, mainly on the fruit fly (Drosophila melanogaster), provide clear examples of genetic assimilation using laboratory populations. The most famous, classical experimental cases of genetic assimilation come from Waddington's work. ${ }^{(2-6)}$ In recent years, modern genetic experiments have confirmed and extended Waddington's early findings. ${ }^{(7,8,24)}$

In 1953, Waddington reported an insightful experiment on the genetic assimilation of the so-called cross-veinless phenotype (cvl), a defect in the wing venation pattern of the fly. ${ }^{(3)}$ The experiment made use of a wild-type population of flies in which, at $25^{\circ} \mathrm{C}$, no $\mathrm{cvl}$ flies were found. Yet, when Waddington exposed pupae of these flies to a heat shock (4 hours at $40^{\circ} \mathrm{C}$, at 17 to 23 hours after puparium formation), some flies exhibited the $\mathrm{cvl}$ phenotype, normally not seen in the population. By heat shocking pupae in every generation and selecting for flies that showed the $\mathrm{cv} /$ phenotype after heat shock ("upward" selection line) or not ("downward" selection line), Waddington created two selection lines. As expected, under such an artificial selection regime, the frequency of the $c v l$ phenotype increased in the "upward" treatment, but decreased in the "downward" treatment, reaching a difference in the proportion of the $\mathrm{cvl}$ phenotype between the treatments of about $60 \%$ after 13 generations of selection. As an internal control for the "upward" selection regime, Waddington also bred flies in each generation, which were not heat shocked as pupae. Remarkably, while he was unable to find any cvl individuals among these untreated control flies until generation 14, after 16 generations the frequency of $\mathrm{cvl}$ started to raise up to $1-2 \%$, despite the absence of a heat shock. Using these flies, Waddington set up paired matings between $\mathrm{cvl}$ individuals, created four selection lines from the offspring of these matings, and obtained a high proportion of $\mathrm{cvl}$ phenotypes at $25^{\circ} \mathrm{C}$ without any heat shock. This classical experiment provides the first clear evidence that an environmentally induced phenotype can be "genetically assimilated".

More recently, genetic assimilation has received empirical support from a study by Gibson and Hogness, ${ }^{(24)}$ who repeated another experiment of Waddington. ${ }^{(4)}$ In their study, the authors selected flies for differential sensitivity to the induction of so-called bithorax phenocopies by ether vapor. ${ }^{(24-}$ 27) Ether-induced bithorax phenotypes resemble (or "phenocopy") genetic bithorax mutants, which have two pairs of wings instead of a single pair. In short, Gibson and Hogness found that the differential phenotypic expression of the etherinduced bithorax phenotype is caused by genetic polymorphisms in the Ubx (Ultrabithorax) gene, with the loss of expression of the UBX protein in the third thoracic imaginal discs correlating with increased sensitivity to ether. Thus, this experiment provides clear evidence that there exists heritable genetic variation for the propensity to exhibit ether-induced bithorax phenotypes; when selecting on this previously cryptic variation, the selected population will eventually lose UBX expression, causing the appearance of a high proportion of bithorax phenotypes in the absence of ether.

In another experiment, Rutherford and Lindquist impaired the function of the chaperone and heat-shock protein HSP90 in flies, either by mutation in the gene encoding HSP90 (Hsp83) or with a specific pharmacological inhibitor, geldanamycin. ${ }^{(7)}$ Impairment of HSP90 function caused a remarkable increase in phenotypic variation, both in laboratory and wild strains. Genetic experiments revealed that these phenotypic variants were caused by several, previously cryptic, genetic determinants. These heritable phenotypic variants could be enriched by selection and rapidly became expressed independently of the enabling mutation; selection caused the continued expression of these traits, even when HSP90 function was restored. These data represent another example of genetic assimilation in the laboratory.

Recent work by Sollars and co-workers takes the notion of assimilation even further. ${ }^{(8)}$ In their experiment, the authors used a nearly totally isogenic Drosophila strain with extremely little genetic variation, carrying a mutant allele for the Krüppel $(K r)$ gene. Flies carrying this mutation have small and rough eyes, the eyes being subject to ectopic outgrowth when flies are fed with the HSP90 inhibitor geldanamycin. Sollars and coworkers kept flies on food containing geldanamycin for a single generation (treatment) or on normal food medium without geldanamycin (control). A single generation of exposure to geldanamycin was necessary to obtain flies with ectopic eye outgrowth; from this population, the authors selected for flies with eye outgrowth in the absence of the drug during 13 generations. In each successive generation, the frequency of the eye outgrowth phenotype increased as compared to the control, from about $1 \%$ in generation 1 to a plateau around $65 \%$ in generation 6 . This plateau was maintained until the experiment was terminated in generation 13. Thus, only with a single exposure to geldanamycin, and with extremely little genetic variation in the population present, the authors "genetically assimilated" the eye outgrowth phenotype by selective breeding. These results suggest that impaired HSP90 function in this population uncovered some previously cryptic, heritable epigenetic variation (i.e. variation in chromatin states) upon which selection could act.

While these experiments do not tell us whether genetic assimilation occurs in natural populations and whether it is an 
adaptive process, they unambiguously demonstrate that genetic assimilation is a plausible mechanism for evolutionary change. In addition to the few cases discussed here, there is a wealth of observations and experimental data in the evolutionary biology literature consistent with the notion of evolution by genetic assimilation. ${ }^{(10,16,19,28-32)}$

\section{First experimental evidence for genetic accommodation of a polyphenism}

While the examples discussed above provide clear cases of genetic accommodation of environmentally insensitive phenotypes (i.e. genetic assimilation), strong and direct experimental evidence for the genetic accommodation of environmentally sensitive traits has so far been lacking. Now, in a Science paper published earlier this year, Suzuki and Nijhout provide the first clear experimental demonstration that evolution of an environmentally sensitive trait, a color polyphenism, can occur by genetic accommodation, at least in the laboratory. ${ }^{(1)}$

Polyphenisms are examples of phenotypic plasticity where a single genotype produces two or more discretely different phenotypes. Such a polyphenism occurs in the hornworm Manduca quinquemaculata, a lepidopteran, which displays larvae of different color. At $20^{\circ} \mathrm{C}$ larvae of this species develop a black phenotype, but at $28^{\circ} \mathrm{C}$ the larvae develop a green phenotype. While the black larval phenotype allows efficient heat absorption during the cold season, the green phenotype is well camouflaged in the environment of the warm season. This apparently adaptive trade-off between thermal regulation and camouflage appears to have driven the evolution of this polyphenism. In their elegant experiment, Suzuki and Nijhout tested whether they could evolve a similar color polyphenism in a related monophenic species, the tobacco hornworm M. sexta, which produces exclusively green larvae. Specifically, the authors aimed at testing whether such a polyphenism could evolve through genetic accommodation, i.e. the genetic stabilization of a stress-induced phenotype.

To reveal previously cryptic, stress-induced phenotypic variants, the authors initially applied a heat-shock treatment to developing larvae of $M$. sexta. However, the larval color remained green, the larvae not showing any sensitivity to this environmental stressor. Next, they repeated the same procedure using a mutant form of $M$. sexta, which exhibits a black larval color. This previously isolated black mutant is known to exhibit reduced levels of juvenile hormone $(\mathrm{JH})$, which causes an increased melanization of the larval cuticle. In contrast to wild type animals, heat-shock treatment of this black mutant resulted in larvae with variable color, ranging from black to green. Suzuki and Nijhout then imposed artificial selection on these different color forms generated in response to the heatshock treatment (Fig. 1A). A polyphenic line was maintained through propagation of individuals that turned greenest after heat shock in each generation. Similarly, as a control, a monophenic line was established by selecting individuals that remained blackest after heat shock.

The response to selection was rapid and strong, indicating that the environmentally induced color variation was heritable. After 13 generations of selection, individuals of the polyphenic line always developed a green phenotype after heat shock. Conversely, individuals of the monophenic line lost the environmental response to heat shock after only 7 generations, remaining black in subsequent generations. In the $13^{\text {th }}$ generation, larvae of both selection lines were examined for their color response to temperatures ranging from $20^{\circ} \mathrm{C}$ to $40^{\circ} \mathrm{C}$ (Fig. 1B). As expected, the monophenic line selected for temperature insensitivity remained black at all temperatures. Selection for the polyphenic line, however, had dramatically changed the color response curve (the so-called reaction norm): at temperatures below $28.5^{\circ} \mathrm{C}$, individuals were mostly black, but at higher temperatures mostly green. Thus, selection of the polyphenic line resulted in a switch-like environmental response to produce two different color forms, that is, a polyphenism. This is a major and very surprising result: although the authors did not directly select for a polyphenic response, for example by selecting individuals in alternating environments (i.e., different temperatures), the color response of the polyphenic line across temperatures changed dramatically during the course of the selection experiment.

In another series of experiments, Suzuki and Nijhout asked which mechanistic changes might underlie the evolution of this polyphenism. An obvious candidate mechanism to examine was regulation by $\mathrm{JH}$ : the black mutant is known to have lower levels of $\mathrm{JH}$ secretion, causing increased melanization of the epidermis, and the sensitive period to heat shock is known to correspond to the $\mathrm{JH}$-sensitive period for color determination. The authors thus first tested whether the polyphenic and monophenic lines differed in the hormonal regulation of melanin synthesis. JH is produced in the corpora allata in the head, and a blood-tight ligature around the larval neck can block hormonal release into the body. Larvae of either the monophenic or the polyphenic line remained black upon such treatment, whether heat shocked or not, suggesting that a cue from the corpora allata, possibly $\mathrm{JH}$, or other cues from the brain, are involved in the temperature-dependent color change. Another experiment used topical application of a synthetic JH analog to larvae and showed that the monophenic line evolved to be less sensitive to $\mathrm{JH}$, whereas the polyphenic line evolved changes in $\mathrm{JH}$ secretion or degradation, but not $\mathrm{JH}$ sensitivity. Finally, JH bioassays revealed JH levels to be higher after heat shock in the polyphenic line as compared to the monophenic line.

Taken together, these results suggest that the experimental evolution of this color polyphenism has occurred through modification of hormonal regulatory mechanisms by genetic

\section{BioEssays 28.9}


A
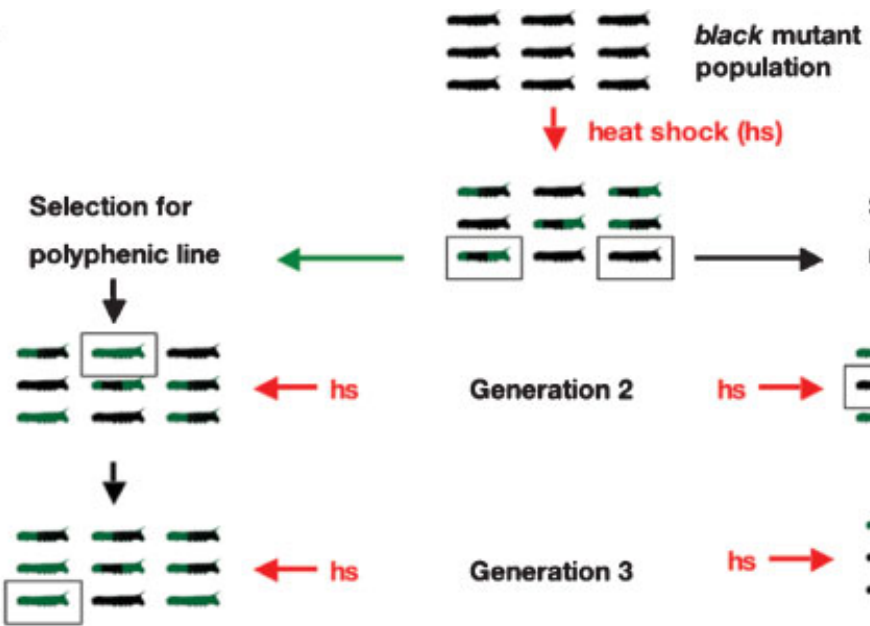

Selection for

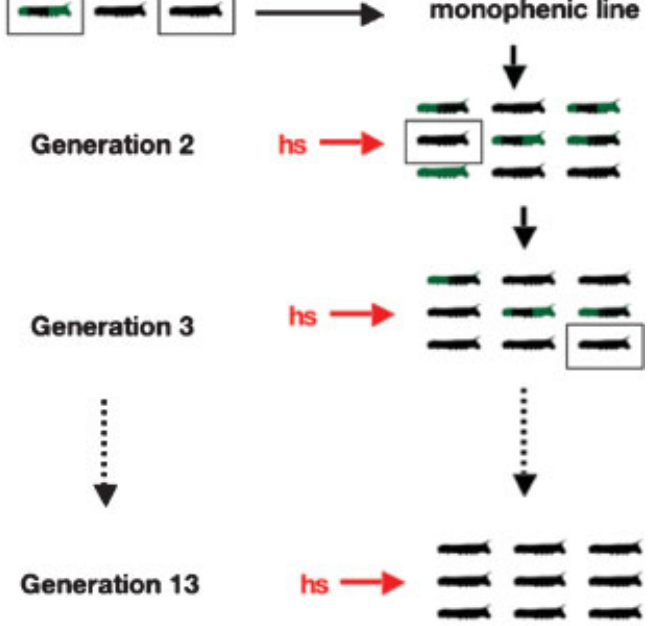

B

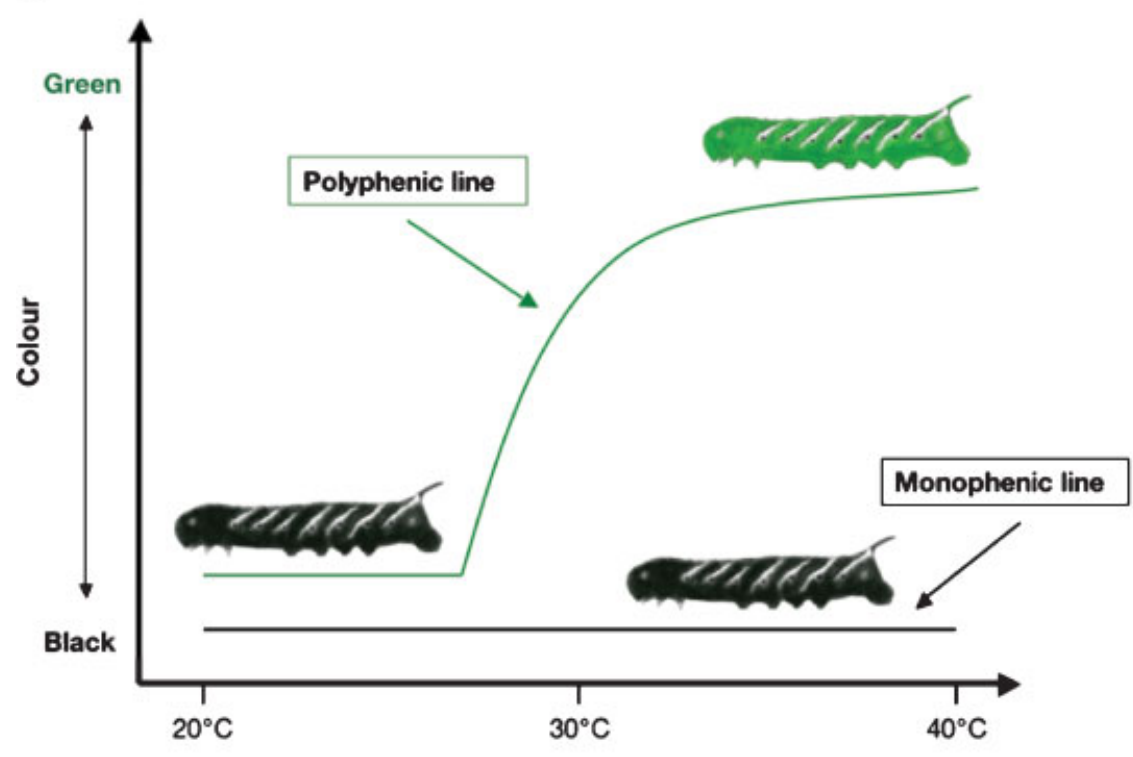

Temperature

Figure 1. A: Schematic outline of the selection experiment by Suzuki and Nijhout. ${ }^{(5)}$ B: Reaction norms of the polyphenic and monophenic line after 13 generations of selection.

accommodation. While the evolution of this polyphenism mimics an adaptive scenario, it currently remains unclear whether the natural polymorphism in M. quinquemaculata has evolved through changes in hormonal regulation. In any case, this study demonstrates that genetic accommodation of a plastic environmental response is possible and may proceed through an interplay of sensitizing mutation, environmental change, and quantitative genetic changes. 


\section{Conclusions}

Understanding how phenotypic variation originates is a major goal of evolutionary biology. Genetic accommodation summarizes the processes of how mutational or environmental inputs can reveal hidden genetic variation for novel phenotypes whose expression subsequently is modified by selection. In our view, there is nothing mysterious about genetic accommodation, as this scenario is entirely compatible with concepts of classical neo-Darwinian evolutionary biology. So why the controversy?

First, the debate is often focused on whether the initial trigger revealing phenotypic variation is environmental or mutational. If the trigger is a mutation, most evolutionary biologists do not have a problem with the scenario of genetic accommodation. In contrast, the idea that environmentally induced change can precede genetic change - for example in the case of genetic assimilation - may cause a headache for people who insist that genetic change has to precede phenotypic change. The aspirin comes in the form of the facts that (1) all the environmental trigger does, is to uncover previously cryptic genetic variation, a well documented phenomenon $^{(17-20)}$ and (2) the response to the environment has to be genetically variable for selection to occur, and thus for genetic accommodation to work. A major biological question about genetic accommodation triggered by environmental inputs, however, remains: how often, in natural populations, are environmental changes sufficiently recurrent and consistent in time so that selection, under such environmental conditions, can lead to the genetic accommodation of the environmentally induced phenotype?

Second, a quite controversial concept related to the concept of genetic accommodation, "adaptive phenotypic accommodation," $(10,33)$ has shifted the attention of many biologists away from the more plausible process of genetic accommodation. This concept postulates adaptive phenotypic adjustments to novel environmental changes or genetic inputs, and assumes that these adaptive adjustments take place with little or no genetic or genomic change and are subsequently genetically accommodated. ${ }^{(10,33)}$ It has been argued that such phenotypic accommodation may be a major factor for the evolution of phenotypic novelties; ${ }^{(10)}$ however, as of yet, it remains unknown to what extent phenotypic accommodation, as envisaged by West-Eberhard, is adaptive and whether it plays a role in evolution.

Third, the somewhat unusual terminology used to describe the concepts of assimilation and accommodation is confusing. For example, the concepts of "assimilation" and "accommodation" are extremely similar — in fact, assimilation is a special case of accommodation, and "genetic assimilation" and "genetic accommodation" in the strict sense refer to nothing but standard evolutionary change of phenotypes and underlying allele frequencies by selection after mutational or environmental changes have uncovered previously cryptic genetic variation. Thus, despite some prevailing semantic and conceptual confusion, both genetic assimilation and genetic accommodation can be phrased entirely in terms of well accepted, standard evolutionary genetic terms such as mutation, environmental change, cryptic genetic variation, sensitivity to genetic change ("genetic variability", see Ref. 20), phenotypic plasticity, threshold traits, and selection. ${ }^{(2-6,9,10,14,16-20)}$ From this perspective, there is nothing exotic about the concepts of genetic accommodation and assimilation.

Most importantly, however, the concept of genetic accommodation has generated a lot of controversy because direct empirical evidence for its evolutionary significance has so far been scarce. Here we have argued that there is indeed solid experimental evidence that genetic accommodation can occur, at least in the laboratory, as illustrated by the various studies on genetic assimilation in Drosophila and by a new experiment on the genetic accommodation of an environmentally sensitive phenotype in Manduca. Yet, whether genetic accommodation is frequent in natural populations and whether it has any adaptive significance remains currently unresolved.

\section{Acknowledgments}

We thank Marie-Anne Félix and Alan O. Bergland for helpful comments on the manuscript.

\section{References}

1. Suzuki Y, Nijhout HF. 2006. Evolution of a polyphenism by genetic accommodation. Science 311:650-652.

2. Waddington $\mathrm{CH}$. 1942. The canalization of development and the inheritance of acquired characters. Nature 150:563.

3. Waddington $\mathrm{CH}$. 1953. Genetic assimilation of an acquired character Evolution 7:118-126

4. Waddington $\mathrm{CH}$. 1956. Genetic assimilation of the bithorax phenotype. Evolution 10:1-13

5. Waddington $\mathrm{CH}$. 1959. Canalization of development and genetic assimilation of acquired characters. Nature 183:1654-1655.

6. Waddington $\mathrm{CH}$. 1961. Genetic assimilation. Adv Genetics 10:257-293.

7. Rutherford SL, Lindquist S. 1998 Hsp90 as a capacitor for morphological evolution. Nature 396:336-342

8. Sollars V, Lu X, Xiao L, Wang X, Garfinkel MD, Ruden DM. 2003. Evidence for an epigenetic mechanism by which Hsp90 acts as a capacitor for morphological evolution. Nature Genet 33:70-74.

9. Masel J. 2004. Genetic assimilation can occur in the absence of selection for the assimilating phenotype, suggesting a role for the canalization heuristic. J Evol Biol 17:1106-1110.

10. West-Eberhard MJ. 2003. Developmental plasticity and evolution. Oxford University Press, Oxford and New York.

11. Williams GC. 1966. Adaptation and natural selection: a critique of some current evolutionary thought. Princeton University Press, Princeton, New Jersey

12. de Jong G, Crozier RH. 2003. A flexible theory of evolution. Nature 424:16.

13. de Jong G. 2005. Evolution of phenotypic plasticity: patterns of plasticity and the emergence of ecotypes. New Phytol 166:101-117.

14. Pigliucci M, Murren CJ, Schlichting CD. 2006. Phenotypic plasticity and evolution by genetic assimilation. J Exp Biol 209:2363-2367.

15. Orr HA. 1999. An evolutionary dead end? Science 285:343-344.

16. Pigliucci M, Murren CJ. 2003. Genetic assimilation and a possible evolutionary paradox: can macroevolution sometimes be so fast as to pass us by? Evolution 57:1455-1464.

17. Gibson, G. \& Dworkin, I. 2004. Uncovering cryptic genetic variation. Nature Rev Genet 5:681-690.

\section{BioEssays 28.9}


18. Dworkin I. 2005a. Canalization, cryptic variation, and developmental buffering: a critical examination and analytical perspective. In Variation-a central concept in biology. (ed. B. Hallgrimsson \& B. K. Hall), pp. 131158: Elsevier (Academic Press)

19. Dworkin I. 2005b. Towards a genetic architecture of cryptic genetic variation and genetic assimilation: The contribution of K.G. Bateman. $J$ Genet 84:223-226.

20. Flatt T. 2005. The evolutionary genetics of canalization. $Q$ Rev Biol 80:287-316

21. Levin DA. 1970. Developmental instability and evolution in periphera isolates. Am Nat 104:343-353.

22. Eshel I, Matessi C. 1998. Canalization, genetic assimilation and preadaptation: a quantitative genetic model. Genetics 149:2119-2133.

23. Pál C, Miklós I. 1999. Epigenetic inheritance, genetic assimilation and speciation. J Theor Biol 200:19-37.

24. Gibson G, Hogness DS. 1996. Effect of polymorphism in the Drosophila regulatory gene Ultrabithorax on homeotic stability. Science 271:200-203.

25. Ho MW, Bolton E, Saunders PT. 1983a. Bithorax phenocopy and pattern formation. I. Spatiotemporal characteristics of the phenocopy response. Exp Cell Biol 51:282-290.
26. Ho MW, Saunders PT, Bolton E. 1983b. Bithorax phenocopy and pattern formation. II. A model of prepattern formation. Exp Cell Biol 51:291-299.

27. Ho MW, Tucker C, Keeley D, Saunders PT. 1983c. Effects of successive generations of ether treatment on penetrance and expression of the bithorax phenocopy in Drosophila melanogaster. J Exp Zool 225:357368.

28. Scharloo W. 1991. Canalization: genetic and developmental aspects. Ann Rev Ecol Syst 22:65-93.

29. Badyaev, AV. 2005. Stress-induced variation in evolution: from behavioural plasticity to genetic assimilation. Proc Roy Soc B 272:877-886.

30. Heil M, Greiner S, Meimberg H, Kruger R, Noyer JL et al. 2004 Evolutionary change from induced to constitutive expression of an indirect plant resistance. Nature 430:205-208.

31. Palmer, AR. 2004. Symmetry breaking and the evolution of development. Science 306:828-833.

32. Grether GF. 2005. Environmental change, phenotypic plasticity, and genetic compensation. Am Nat 166:E115-E123.

33. West-Eberhard MJ. 2005. Phenotypic accommodation: adaptive innovation due to developmental plasticity. J Experimental Zool (Mol Dev Evol) 304B:610-618. 